\title{
O uso do ATLS nos complexos traumas de artéria axilar, vencendo esse desafio - relato de caso e revisão da literatura
}

\author{
The use of ATLS in complex axillary artery trauma. Overcoming this challenge. \\ Case report and literature review
}

\author{
Matheus Polly $^{1}$, Marília Arrais ${ }^{1}$, Bruna Queiroz Coelho ${ }^{1}$, Gabriela Tognini Saba ${ }^{1}$, \\ Rafael Fürst' ${ }^{2}$, Afonso César Polimonti ${ }^{2}$, João Antonio Correa ${ }^{3}$
}

Polly M, Arrais M, Coelho BQ, Saba GT, Fürst R, Polimonti AC, Correa JA. O uso do ATLS nos complexos traumas de artéria axilar, vencendo esse desafio - relato de caso e revisão da literatura / The use of ATLS in complex axillary artery trauma. Overcoming this challenge. Case report and literature review. Rev Med (São Paulo). 2014 out.-dez.;93(4):165-71.

RESUMO: INTRODUÇAO: O trauma das artérias subclávia e axilar é incomum, mas potencialmente grave. Os ferimentos penetrantes por projétil de arma de fogo e por arma branca constituem o mecanismo habitual. A complexidade anatômica torna desafiador o tratamento destas lesões. O seguimento adequado do protocolo ATLS é o principal responsável pelo bom prognóstico nos traumas de grande potencial de mortalidade como os traumas arteriais dessa topografia. $\mathrm{O}$ acesso para abordagem da artéria axilar depende da extensão e localização da lesão. Na maior parte dos casos, uma incisão supra e/ou infraclavicular é suficiente para a abordagem entretanto o controle proximal da artéria subclávia pode ser necessário. Nesse caso, o acesso cirúrgico geralmente envolve a esternotomia mediana - para lesões do lado direito - e a toracotomia ântero- lateral para lesões do lado esquerdo. Neste contexto, o trauma da artéria axilar é uma lesão desafiadora. Relata-se um caso singular que recebeu tratamento inicial de acordo com o protocolo ATLS com ligadura da artéria axilar. Métodos/Paciente: Este trabalho analisa a conduta cirúrgica e de trauma de um paciente do sexo masculino, 23 anos que deu entrada com ferimento por arma de fogo em topografia infra-clavicular esquerda e choque hipovolêmico. $\mathrm{Na}$ drenagem pleural houve saída de mais de $2000 \mathrm{ml}$ de sangue submetendo-o à toracotomia e ligadura de artéria axilar. Foi encaminhado para serviço de atenção terciária e realizada a revascularização com enxerto de veia safena. RESULTADOS: A conduta de atendimento ao Trauma pelo ATLS foi realizada de maneira correta, com a rápida identificação de lesão grave com potencial elevado de morbimortalidade e necessidade de transferência que permitiu a revascularização do paciente alcançando um ótimo prognóstico com sobrevivência do paciente e manutenção da função do membro. CONCLUSÃO: As lesões de artéria axilar são graves, com potencial letal elevado e necessitam de um tratamento rápido e bem planejado. O paciente deve receber o tratamento inicial pelo protocolo ATLS com estabilização do quadro e posterior transferência tratamento definitivo se necessário.

DESCRITORES: Traumatismos torácico; Toracotomia; Cuidados de suporte avançado de vida no trauma; Ferimentos penetrantes; Artéria axilar, Relatos de casos; Literatura de revisão como assunto.

ABSTRACT: The trauma of the subclavian and axillary arteries is uncommon but potentially serious. Penetrating injuries by firearm bullet and stabing are their usual mechanism. The anatomical complexity makes these injuries challenging to treat. Strictly following the ATLS protocol is the primarily responsible for the

1. Acadêmicos de Medicina, Faculdade de Medicina do ABC - FMABC, Santo André, SP.

2. Professor Adjunto da Cirurgia Vascular da Faculdade de Medicina do ABC - FMABC, Santo André, SP.

3. Professor Titular da Disciplina de Angiologia e Cirurgia Vascular da Faculdade de Medicina do ABC - FMABC, Santo André, SP.

Endereço para correspondência: Matheus Polly. Rua Dinah Silveira de Queiroz, 526. City América, São Paulo, SP. CEP: 05119-090.

E-mails: matheus.fmabc@gmail.com, brunaqccoelho@hotmail.com,cor.jantonio@gmail.com 
good prognosis in the high mortality potential that are arterial trauma in that topography. Access to approach the axillary artery depends on the extent and location of the lesion. In most cases a supra and / or infraclavicular incision are sufficient for the proximal control of the bleeding, however proximal subclavian artery ligature may be required. This cases generally involves the surgical access by median sternotomy - injuries to the right - and anterolateral thoracotomy for left lesions. In this context, axillary artery injury is a challenging trauma. We report a unique case that received initial treatment according to the ATLS protocol with ligation of the axillary artery. This paper analyzes the trauma and surgical approach of a 23 years old male lodged injuried by firearm in the left infra-clavicular topography presenting hypovolemic shock. In pleural drainage was out of more than $2000 \mathrm{ml}$ of blood by subjecting it to thoracotomy and axillary artery ligation. He

\section{INTRODUÇÃO}

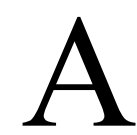
s lesões de artéria axilar (AA) e subclávia (ASC) são manifestações vasculares raras, constituindo aproximadamente de $2 \%$ a $3 \%$ das lesões vasculares traumáticas. Apesar de sua baixa prevalência é de extremo interesse médico por seu alto nível de complexidade, elevado potencial letal e mórbido. Sua etiologia é variada sendo lesões derivadas de violência o mecanismo mais comum, com ferimentos por arma de fogo como principal causa seguido por ferimento por arma de branca, sendo está a apresentação menos grave ${ }^{1-3}$.

O Advanced Trauma Life Suppor $\mathrm{B}(\mathrm{ATLS} \AA$ ) é um protocolo criado em 1976 que sistematizou o atendimento ao paciente traumatizado; seu advento permitiu uma análise mais objetiva dos casos de trauma, possibilitando o rápido diagnóstico de alterações com alto risco de morte e seu rápido tratamento, de maneira organizada e completa. O ATLS ${ }^{\circledR}$ é o grande responsável pelo aumento de sobrevida de pacientes traumatizados nas últimas décadas. Com a padronização para o atendimento de traumas torácicos com de lesões de AA e ASC o prognóstico é extremamente favorável para estes pacientes quando rapidamente avaliados e tratados, geralmente evoluindo com sobrevivência e sem sequelas do trauma. Se não forem rapidamente encaminhados para um centro de trauma, as lesões arteriais de AA e ASC tem mortalidade pré-hospitalar variável em 30-75\% dos pacientes ${ }^{1}$.

A experiência clínica limitada, anatomia complexa e dificuldade de acesso à topografia afetada tornam as lesões de AA e ASC um desafio para os médicos que lidam com pacientes traumatizados. $\mathrm{O}$ grau de lesão varia de acordo com o mecanismo de trauma. Atualmente lesões parciais das artérias AA e ASC podem ser tratadas por técnicas endovasculares, que geram muito menos complicações no pós operatório; porém, em casos graves, como transfixação das artérias ou sangramentos maciços, uma abordagem direta faz-se necessária ${ }^{3,4}$. was referred to tertiary care service and held revascularization with saphenous vein graft. The conduct of care Trauma by ATLS was performed correctly, with the rapid identification of serious injury with a high potential for morbidity and mortality and need to transfer that allowed revascularization of patients achieving an excellent prognosis with patient survival and limb function maintenance. Thus, the axillary artery injuries are severe, with high lethal potential and need a fast and well-planned treatment. The patient should receive initial treatment by ATLS protocol, receiving life suport mesuares for its stabilization and subsequent transfer to definitive treatment if necessary.

KEYWORDS: Thoracic trauma; Thoracotomy; Advanced trauma life support care; Wounds, penetrating; Axillary artery; Case reports; Review literature as topic.

$\mathrm{O}$ acesso às artérias AA e ASC é complicado e o local da lesão e estabilidade hemodinâmica do paciente vão nortear a escolha do melhor acesso cirúrgico ${ }^{3}$. Pacientes hemodinamicamente estáveis devem ter suas artérias estudadas antes da realização de reparo; técnicas que podem ser utilizadas são a angiotomografia - exame de escolha - ou a arteriografia; geralmente esses exames dão subsídio para o planejamento do acesso cirúrgico, que pode ser uma incisão infra-clavicular com ou sem luxação

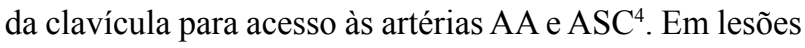
graves, em pacientes hemodinamicamente instáveis ou com critérios de indicação, deve-se abordar as artérias diretamente para controle hemorrágico, para isso realizase a esternotomia medial ou a toracotomia antero-lateral; em relação as duas técnicas muito se discute sobre qual a melhor abordagem de acordo com o lado da lesão e complicações de pós-operatório3 ${ }^{3}$.

O tratamento definitivo das lesões de AA e ASC dependem da extensão da lesão e da abordagem realizada no controle da hemorragia. Em lesões completas das artérias podem ser realizadas a rafia primária da artéria, enxerto autólogo com veia periférica e próteses vasculares.

Com o aumento da violência urbana é crescente o número de traumas torácicos perfurantes que podem afetar os grandes vasos, assim discutir as lesões de AA e ASC é importante para a conscientização dessa manifestação complexa que deve ser tratada como urgência e da maneira adequada. Este trabalho traz um relato de experiência e de um paciente com lesão em AA que teve excelente evolução pelo seguimento da conduta proposta no $A T L S \circledR$, demonstrando técnicas de estabilização e tratamento definitivo que permitiram sua evolução sem sequelas no pós-operatório.

\section{MÉTODOS E PACIENTE}

Para esse estudo será considerado o relato de experiência de um paciente que sofreu um trauma vascular 
de topografia axilar e os procedimentos realizados em seu atendimento. Foram levantadas informações gerais sobre o paciente, mecanismo de trauma, local de atendimento, medidas iniciais de suporte de acordo com o protocolo ATLS, medidas de estabilização, conduta para encaminhamento para centro terciário, tratamento definitivo das lesões (técnica cirúrgica) e evolução pós operatória. Também, realizou-se uma revisão de literatura nas bases de dados PubMed e SciELO com os termos: Lesão de Artéria Axilar, Lesão de Artéria Subclávia, Toracotomia/Emergência, Lesão Vascular dos Membros, Tratamento de lesões vasculares e Lesões penetrantes do Tórax, selecionando-se artigos relevantes para realização de discussão frente à conduta e resultados alcançados.

\section{RELATO DE CASO}

Paciente de 23 anos do sexo masculino dá entrada no pronto socorro de um serviço secundário de atenção, trazido pelo resgate em prancha rígida com colar cervical e "Head Block" e com acesso venoso periférico com bolsa de ringer lactato em infusão contínua, vítima de ferimento por arma de fogo de baixa velocidade (Pistola de Mão/ Revolver) em região infra-clavicular esquerda. Na entrada iniciou-se o protocolo padronizado pelo ATLS, no exame primário o paciente encontrava-se com:

A: Vias Aéreas Pérvias com colar cervical;

B: Avaliação: Taquipnéico, com expansibilidade diminuída em hemitórax esquerdo e normal à direita, percussão maciça em base e médio hemitórax esquerdo com abolição de Múrmurio à ausculta do mesmo lado, em ápice de pulmão esquerdo som claro pulmonar, à direita percussão claro pulmonar de ápice à base com murmúrio vesicular presente globalmente. Nota-se lesão perfurocontusa em topografia infraclavicular esquerda;

C: Conduta: Ofereceu-se oxigênio em alta concentração por máscara;

D: Avaliação: Sem sinais de hemorragia externa importante. Pelo exame físico adquirido em B alto risco de hemorragia intrapleural. Pele descorada com pulsos finos, rápidos e regulares;

E: Conduta: Inserção de outro acesso venoso periférico com cateter calibroso com obtenção de amostra de sangue para tipagem e prova cruzada, iniciou-se infusão contínua de outra bolsa de ringer lactato de 1 litro;

F: Avaliação: Paciente encontra-se com 15 pontos na escala de coma de Glasgow, com pupilas isocóricas fotorreagente;

G: Paciente é completamente despido e coberto com manta térmica para evitar hipotermia.

Ao mesmo tempo, o paciente é colocado em monitorização com oximetria de pulso, eletrocardiografia e são passadas sondas Nasogástrica e Uretral para controle de diurese.

A equipe responsável pelo atendimento prosseguiu o atendimento com a drenagem do tórax com torascostomia por tubo torácico em selo de d'água. No momento da drenagem há saída de mais de $2000 \mathrm{ml}$ de sangue pelo tubo. Solicita-se à central de regulação do serviço a busca de vaga em serviço terciário para transferência após procedimentos cirúrgicos para controle de hemorragia.

Na sala de trauma o paciente é sedado e entubado e, de acordo com a indicação e topografia optasse pela realização de toracotomia antero-lateral. Durante a inspeção da caixa torácica percebe-se a transfixação da artéria axilar esquerda como fonte principal de hemorragia. Como conduta de resgate e controle de danos a equipe cirúrgica amarra a artéria subclávia proximalmente cessando a hemorragia intra-torácica (Figura 1)

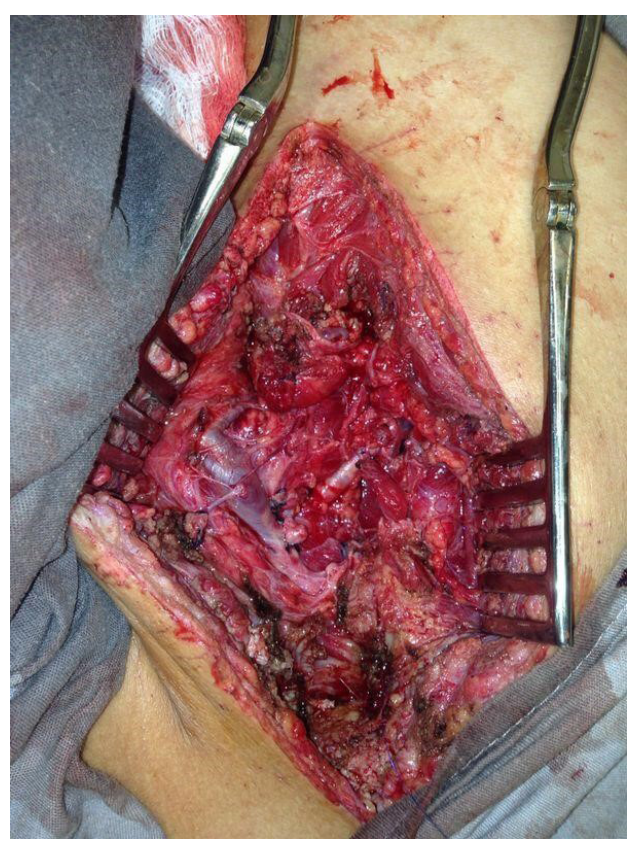

Figura 1: Artéria sub-clávia amarrada em sua origem

Após controle hemorrágico e estabilização do paciente é fechada a toracotomia e solicitada a remoção para o serviço terciário de referência após período pós-cirúrgico para tratamento vascular definitivo rápido para preservação da função do membro superior esquerdo que encontra-se privado de seu suprimento sanguíneo arterial. No serviço terciário o paciente dá entrada sedado e entubado e é avaliado pela equipe de emergência e de cirurgia vascular (Figura 2) que solicita uma sala de cirurgia e prepara o paciente para a revascularização.

No centro cirúrgico, após tomas as devidas medidas de anti-sepsia e sedação, reaborda-se a toracotomia do paciente com dissecação cuidadosa do plexo arterial subclávio-axilar. Frente a lesão total da artéria com amarração proximal da subclávia, a conduta escolhida é a revascularização com enxerto autólogo de veia safena magna. É realizada a dissecção cuidadosa de 
uma das safenas do paciente com posterior anastomose e restabelecimento de fluxo para o membro superior esquerdo. O procedimento seguiu sem complicações e no pós operatório institui-se antibiótico terapia profilática pelo mecanismo contaminado da lesão e risco de infecção do enxerto e cavidade torácica. Na Figura 3 tem-se o resultado da revascularização.

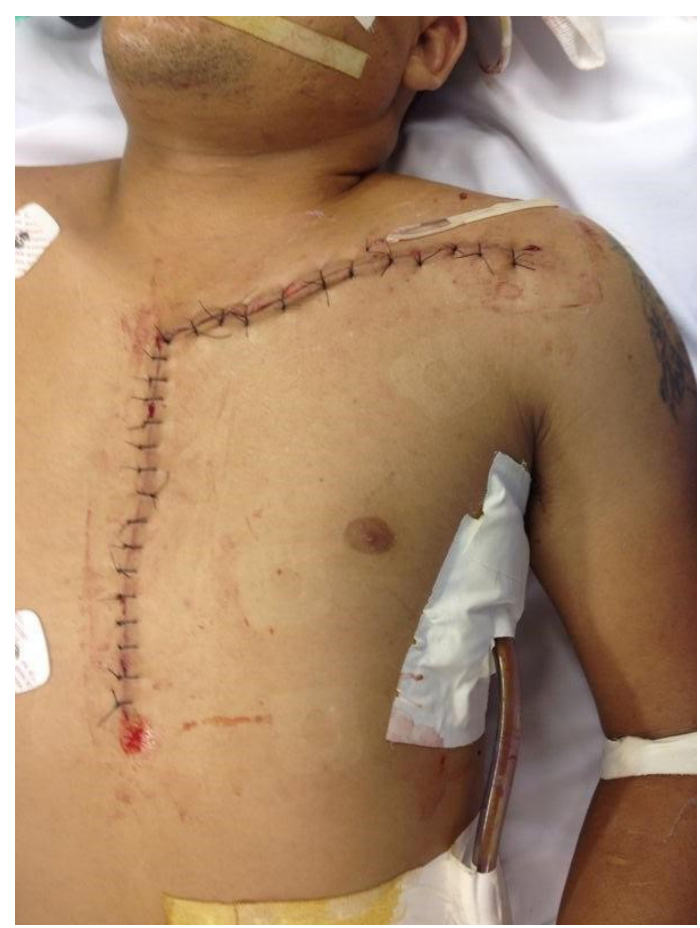

Figura 2 - Aspecto de entrada ao serviço terciário

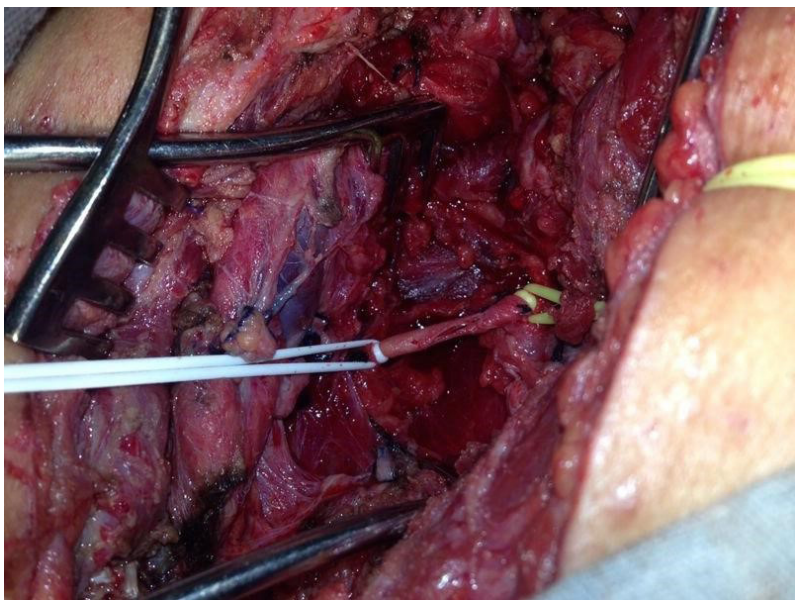

Figura 3 - Resultado da revascularização de artéria sub-clávia com veia safena magna

\section{RESULTADOS}

$\mathrm{Na}$ pesquisa para realização da discussão foram selecionados 17 artigos associados aos temas de maior relevância na discussão do caso apresentado.

Em relação à experiência cirúrgica do caso apresentado houve uso correto e consciente do protocolo ATLS, com exame primário realizado rapidamente, identificação de lesão grave, interpretação da necessidade de encaminhamento, manejo inicial para estabilização e controle de danos com posterior remoção para centro terciário para tratamento definitivo.

O paciente evoluí sem complicações no pós-operatório com função completa do membro sem qualquer déficit neuromotor associado.

\section{DISCUSSÃO}

Lesões das artérias AA e ASC são graves e continuam a ser um grande desafio cirúrgico. A severidade do trauma está diretamente ligada ao mecanismo, sendo ferimentos causados por arma de fogo os mais importantes. Aproximadamente de $60-75 \%$ dos pacientes com esse tipo de lesão são vítimas de ferimento por arma de fogo, o que está associado a maior mortalidade pré-hospitalar e pior prognóstico no tratamento, levando ainda a um pior índice de complicações cirúrgicas pela necessidade de acessos maiores para correção das lesões. A mortalidade geral de pacientes com lesões nesta topografia tratados é variável de 5\%-30\% de acordo com o serviço estudado ${ }^{1-4}$.

A artéria axilar é uma continuação da artéria subclávia e é um calibroso vaso que recebe sangue em alta pressão sendo ramo quase direto do arco aórtico. O seu início é localizado na margem lateral da primeira costela e o seu fim, na margem inferior do músculo redondo maior, onde ela continuará como artéria braquial. A AA é divida em três partes, tomando-se como referência o músculo peitoral menor. A primeira parte da AA está situada entre a margem lateral da primeira costela e a margem medial do músculo peitoral menor. A segunda parte se encontra posteriormente ao $\mathrm{m}$. peitoral menor. A terceira parte se estende da margem lateral do músculo peitoral menor até a margem inferior do músculo redondo maior. Existem quatro ramos arteriais cuja origem é a AA: artéria torácica superior, artéria toracoacromial, artéria torácica lateral e artéria subescapular. Juntas, essas artérias conseguem suprir as seguintes estruturas anatômicas: músculos peitorais, músculo subclávio, músculo serrátil anterior, músculo intercostal, linfonodos axilares e face lateral da mama ${ }^{5}$. Sua descrição anatômica demonstra a intensa trama vascular associada à topografia, que leva a volumosa hemorragia em sua lesão. Também, devido a grande vascularização local é frequente o número de pacientes - aproximadamente $80 \%$ - que não apresentam sinais de isquemia precoce no membro do lado afetado. Os principais sintomas são parestesia do membro e diminuição/ abolição do pulso ${ }^{1}$.

A utilização do ATLS $®$ é o principal fator de bom prognóstico nos casos de lesão torácica traumática, e em traumas no geral. Neste caso, o manejo correto desse instrumento foi o responsável pela sobrevivência e boa evo- 
lução do caso. Por permitir uma rápida avaliação e estratificação de risco, o ATLS ${ }^{\circledR}$ permite a indicação precoce de tratamentos que podem salvar a vida do paciente crítico, bem como institui medidas de suporte que colaboram para o bom prognóstico ${ }^{6}$.

Nos traumas torácicos em geral entre $15-30 \%$ dos pacientes necessitam de algum tipo de toracotomia para o reparo de lesões intra-torácicas. Esse número, quando se trata de lesões de AA e ASC, é muito maior variando de $80 \%-90 \%$ dos $\operatorname{casos}^{1-5,7,8}$. Apenas nas lesões parciais em pacientes hemodinamicamente estáveis o tratamento endovascular pode ser realizado e a toracotomia é dispensável $^{5}$. Um estudo americano padronizou o atendimento das lesões de AA e SCV para a triagem de tratamento de suas lesões. O algoritmo (Figura 4) mostra que pacientes estáveis com sinais de lesão vascular descoberto em exame clínico/drenagem de tórax, ou pacientes com sinais radiológicos de lesão arterial devem ser submetidos à arteriografia diagnóstica (Figura 5) para avaliar a extensão do ferimento. Caso a lesão seja passível de reparo endovascular, este deve ser o método escolhido ${ }^{5}$.

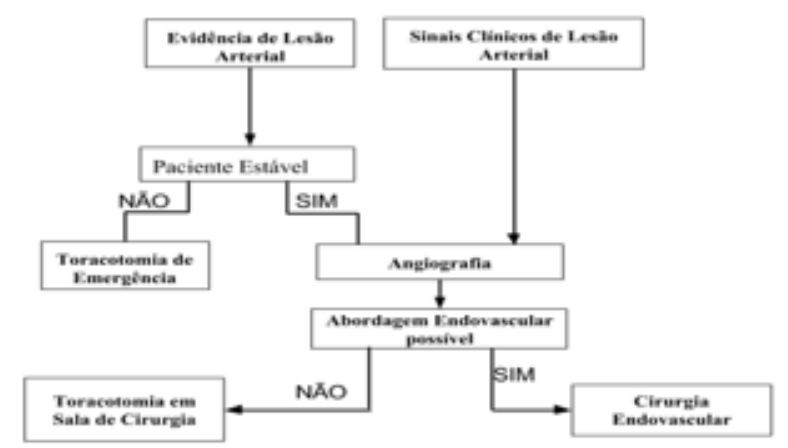

Figura 4 - Algoritmo para tratamento endovascular de lesões arteriais $^{5}$

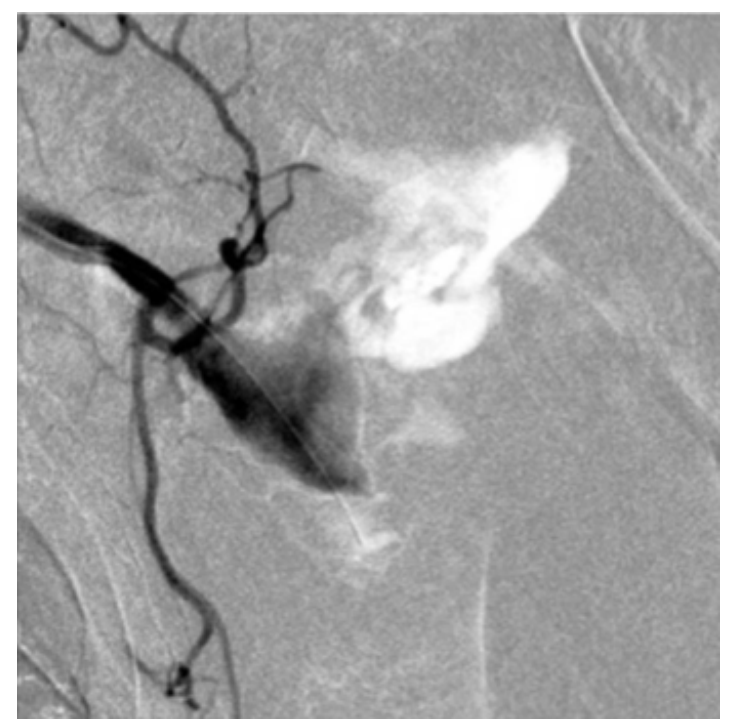

Figura 5 - Angiografia demonstrando a ruptura de artéria axilar e extravasamento de contraste ${ }^{8}$
A toracotomia na sala de emergência é um procedimento com alta mortalidade - de 73\%-100\% com índice de complicação pós-operatória de 36\%. Por isso deve ser bem indicada e realizada por profissional capacitado e experiente no manejo deve tipo de abordagem. As principais indicações para toracotomia de emergência são choque hipovolêmico profundo, parada cardíaca associada a trauma torácico e controle de hemorragia intra-torácica, evidenciada pelo hemotórax maciço que é definido como a saída maior que $1500 \mathrm{ml}$ de sangue na drenagem pleural. Existem alguns objetivos que podem ser alcançados com o a toracotomia na sala de emergência: controle de hemorragia, compressões cardíacas efetivas, clampeamento do hilo pulmonar, drenagem direta de tamponamento cardíaco e clampeamento transverso da aorta descendente. É consenso que a seleção deve haver rígida seleção de pacientes que podem se beneficiar da toracotomia em sala de emergência sendo os que tem melhor prognóstico aqueles com trauma torácico isolado, lesão única, presença de sinais vitais na admissão e lesão por arma branca ${ }^{6,7}$.

A dificuldade de exposição da AA e ASC é um desafio para a maioria dos cirurgiões de trauma. O controle da hemorragia por compressão pode não ser possível pela localização retroclavicular da AA e ASC, um método para controle provisório é a passagem de uma sonda balonada através de uma incisão supra-clavicular com enchimento do balão a nível do feixe vascular da sub-clávia. Essa técnica pode adiar a necessidade de toracotomia na sala de emergência possibilitando a transferência do paciente para a sala de cirurgia. Toracotomias realizadas em sala de cirurgia tem um melhor prognóstico e menor risco de complicações - sendo o risco imediato a mediastinite infecciosa ${ }^{2,9}$.

A realização de um acesso cirúrgico adequado é essencial na abordagem dos traumas de AA e ASC. Em pacientes estáveis sem hemotórax maciço uma incisão supraclavicular com deslocamento da clavícula pode ser o suficiente para a abordagem da lesão; porém, em lesões proximais o acesso por toracotomia faz-se necessária. De maneira geral o acesso em lesões ao lado direto do hemitórax são abordadas com a associação de uma esternotomia medial com incisão clavicular, enquanto do lado esquerdo a melhor abordagem é a toracotomia anterolateral em "livro aberto", que expõe completamente o arco aórtico e os grandes vasos. Nesta experiência foi optado pela toracotomia antero-lateral que possibilitou a visualização da lesão e controle proximal da transfixação da artéria axilar ${ }^{2,6,10,11}$. Há referências que demonstram que a utilização de esternotomia mediana é suficiente em ambos os lados, e que esse procedimento tem menor potencial mórbido sendo assim mais indicado (Figura 6). 


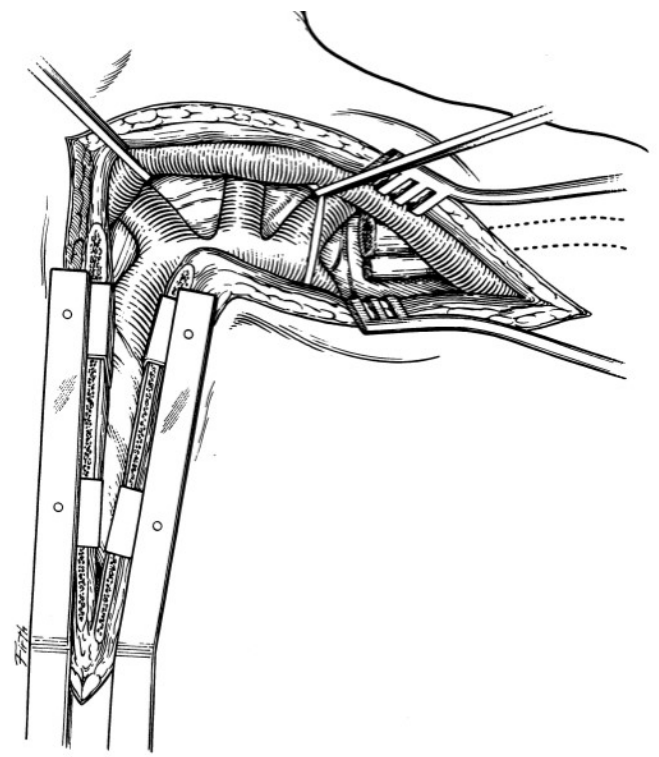

Figura 6 - Esternotomia medial combinada com incisão supra clavicular a esquerda ${ }^{9}$

O dano venoso é associado na quase totalidade das lesões de AA e ASC, evidências demonstram que a ligadura das veias lesadas é o tratamento de escolha o que não acarreta em maiores complicações ou perda funcional para o paciente, que pode evoluir com edema do membro afetado no pós-operatório ${ }^{1,2,8}$. A perda de função do membro superior está associada à lesão do plexo braquial nos traumas perfurantes que atingem essa região. A perda de função do membro não é comum, sendo esperada a recuperação total de sensibilidade e mobilidade quando há o correto manejo dos traumas da AA e ASC.

O tratamento realizado por cirurgiões de trauma, quando comparado ao cirurgião vascular, não mostrou diferenças em prognóstico, sendo que o principal fator é o manejo rápido da hemorragia. Porém, nota-se um número menor de complicações pós-operatórias quando os procedimentos são realizados por cirurgião vascular ${ }^{12}$.

O tratamento definitivo das lesões de AA e ASC que necessitam de controle de hemorragia com ligação proximal artéria varia de acordo com o mecanismo de lesão. Em ferimentos por arma branca pode-se tentar a rafia primária, que cursa com maior número de complicações e necessidade de reabordagem, ou realização de enxertos, que podem ser autólogos venosos, como o utilizado nessa experiência ou com próteses vasculares ${ }^{2,12}$. O uso de próteses vasculares está associado ao risco, apesar que incomum, de infecção. Considerando a natureza contaminada dos traumas penetrantes torácicos por arma de fogo, seu uso deve ser feito com cuidado, uma fez que o diagnóstico de infecção de prótese vascular, cujo principal agente causador é o $S$. aureus, é difícil e traz um grave risco associado 9 .

\section{CONCLUSÃO}

Os traumas de AA e ASC são lesões graves que devem ser tratadas com prontidão com alto potencial de mortalidade se não tratados precocemente. Assim, o uso do ATLS ${ }^{\circledR}$ mostra-se essencial na sobrevivência de pacientes com lesões graves dessa topografia, pois permite uma rápida triagem e diagnóstico das lesões, bem como indica o tratamento e medidas de suporte necessárias para a estabilização do paciente. Seu uso correto foi o grande diferencial para a sobrevivência do paciente aqui relatado. Ainda, este protocola a necessidade de busca ao serviço terciário para um atendimento com mais nível de atenção ou tratamento definitivo, que neste caso permitiram o tratamento definitivo da lesão possibilitando a total recuperação do paciente. As abordagens às artérias AA e ASC dependem do local e extensão da lesão, sendo a incisão supraclavicular, a esternotomia medial combinada à anterior e a toracotomia antero-lateral em "livro aberto" as possibilidades de acesso. O uso da toracotomia anterolateral está associado a maiores complicações enquanto a esternotomia mostra-se um procedimento viável independente do lado acometido. O profissional que realiza esses procedimentos deve ser qualificado e deve selecionar aqueles que se beneficiarão de tais abordagens, devido à alta taxa de mortalidade e complicações; não houve diferenças significativas nos tratamentos por cirurgiões vasculares e de trauma, sendo o principal fator prognóstico o tempo de tratamento, assim, havendo profissional habilitado o tratamento não deve ser adiado. Quando não houver critérios para toracotomia, porém sinais de lesão arterial torácica, o exame diagnóstico padrão ouro é a angiotomografia computadorizada, que pode ser substituída pela arteriografia. São instrumentos importantes na avaliação da extensão da lesão arterial e preditores de tratamento. Atualmente o tratamento endovascular vem ganhando espaço como tratamento definitivo de lesões arteriais dessa topografia, uma vez que traz menos dano cirúrgico e complicações para o paciente, porém, devido a alta complexidade dos traumas dessa topografia seu estudo é de extrema importância uma vez que o tratamento adequado dessas lesões é o diferencial no bom prognóstico do paciente. Essa experiência cirúrgica é rica em demonstrar o valor do correto uso do ATLS $₫$, que mesmo em lesões raras e de alta mortalidade, quando aplicado propicia ao médico grande segurança no manejo de seu paciente, permitindo um tratamento completo e principalmente garantindo a sua sobrevivência.

\section{REFERÊNCIAS}

1. Sobnach S, Nicol AJ, Nathire H, Edu S, Kahn D, Navsaria DH. An analysis of 50 surgically managed penetrating subclavian artery injuries. Eur J Vasc Endovasc Surg. 2010;39(2):155-9. doi: 10.1016/j.ejvs.2009.10.013. 
2. Lin PH, Koffron AJ, Guske PJ, Lujan HJ, Heilizer TJ, Yario RF, Tatooles CJ. Penetrating injuries of the subclavian artery. Am J Surg. 2003;185:580-4. doi:10.1016/S00029610(03)00070-9.

3. Demetriades D, Chahwan S, Gomez H, Peng R, Velmahos G, Murray J, Asensio J, Bongard F. Penetrating injuries to subclavian and axillary vessels. J Am Coll Surg. 1999;188(3):290-5. http://dx.doi.org/10.1016/S1072-7515 (98)00289-0.

4. Carrick MM, Morrison CA, Pham HQ, Norman MA, Marvin B, Lee J, Wall MJ Jr, Mattox KL. Modern management of traumatic subclavian artery injuries: a single institution's experience in the evolution of endovascular repair. Am J Surg. 2010;199(1):28-34. doi: 10.1016/j.amjsurg.2008.11.031.

5. Moore KL. Membro superior. In: Moore KL, Dalley AF, Agur AMR, editores. Anatomia orientada para clínica. Rio de Janeiro: Guanabara Koogan; 2011. p.665-813.

6. American College of Surgeons. Advanced trauma life support ${ }^{\circledR}$ student course manual. Chicago: ACS Committee on Trauma; 2012.

7. Karmy-Jones R, Nathens A, Jurkovich GJ, Shatz DV, Brundage S, Wall MJ Jr, Engelhardt S, Hoyt DB, Holcroft J, Knudson MM, Michaels A, Long W.
Urgent and emergent thoracotomy for penetrating chest trauma. J Trauma. 2004;56(3):664-9. doi: 10.1097/01. TA.0000068238.74552.4B.

8. Ergunes K, Yazman S, Yetkin U, Cakır V, Gurbuz A. Axillary artery transection after shoulder dislocation. Ann Vasc Surg. 2013;27(7):974.e7-10. doi: 10.1016/j.avsg.2013.04.002.

9. Lichtenfels E, Frankini AD, Cardozo MA, D'Azevedo PA. Infecção em endoprótese. J Vasc Bras. 2011;10(1):504. Disponível em: http://www.scielo.br/pdf/jvb/v10n1/ v10n1a09.

10. Westphal FL, Lima LC, Lima Neto JC, Silva JS, Santos Júnior VL, Westphal DC. Trauma torácico: análise de 124 pacientes submetidos à toracotomia em Manaus. Rev Col Bras Cir. 2009;36(6):482-6. http://dx.doi.org/10.1590/ S0100-69912009000600004.

11. Spencer Netto FAC, Campos JM, Lima LFC, Rivieira MACP, Kreimer F, Silveira RK. Fatores prognósticos de mortalidade em pacientes com trauma cardíaco que chegam à sala de cirurgia. Rev Col Bras Cir. 2000;28(2):87-94. Disponível em: http://www.scielo.br/pdf/rcbc/v28n2/02.pdf.

12. Krüger A, Florido C, Braunisch A, Walther E, Yilmaz TH, Doll D. Penetrating arterial trauma to the limbs: outcome of a modified protocol. World J Emerg Surg. 2013;8(1):51. doi: 10.1186/1749-7922-8-51. 